\title{
Schizophrenia: inorganic no more
}

\section{Michael A P Bloomfield PhD ${ }^{1,2}$, S Caroline Buck MBChB ${ }^{2}$, *Oliver D Howes DM ${ }^{1,3}$}

${ }^{1}$ Psychiatric Imaging Group, MRC Clinical Sciences Centre, Institute of Clinical Sciences, Hammersmith Hospital, Imperial College London, Du Cane Road, London W12 0NN. United Kingdom

${ }^{2}$ Division of Psychiatry, University College London, Maple House, 149 Tottenham Court Road, London WC1T 7NF. United Kingdom

${ }^{3}$ Department of Psychosis Studies, Institute of Psychiatry, Psychology \& Neuroscience, Kings College London, De Crespigny Park, London SE5 8AF. United Kingdom

*Author to whom correspondence should be addressed:

Dr Oliver D Howes

Psychiatric Imaging Group

MRC Clinical Sciences Centre

Hammersmith Hospital

Du Cane Road

London W12 0NN

United Kingdom

Email: $\quad$ oliver.howes@csc.mrc.ac.uk

Telephone: $\quad$ +44(0)2083833446

The authors confirm that the paper has not been submitted to another journal, and has not been published in whole or in part elsewhere previously. 
Schizophrenia is a mental illness affecting millions of people worldwide and is characterised by "fundamental and characteristic distortions of thinking and perception" (ICD) ${ }^{1}$. The international diagnostic criteria classify schizophrenia as a non-organic disorder, at the same clinicians continue to use "organic" in day-to-day practice to refer to non-"functional" causes of psychosis and vice versa. This is despite mounting evidence to the contrary and has important implications for our treatment of patients with schizophrenia and resource allocation.

In the medical context, the term organic is generally taken to mean an illness in which there is change in the structure or function of an organ. In addition, the ICD-10 describes several criteria to differentiate between nonorganic and organic disorders (table). 


\begin{tabular}{|c|c|}
\hline $\begin{array}{l}\text { ICD-10 Criteria supporting a diagnosis of organic } \\
\text { disorder }^{1}\end{array}$ & Key findings in schizophrenia \\
\hline Evidence of cerebral disease, damage or dysfunction. & $\begin{array}{l}\text { Increase in lateral ventricle size }^{2} \text {. } \\
\text { Reduced cross-sectional area of the corpus callosum }{ }^{3} \text {. } \\
\text { Reduced volumes of the temporal structures }{ }^{3} \text {. } \\
\text { Reduced fronto-temporal white matter connectivity }{ }^{4} \text {. } \\
\text { Elevated microglial cell density post } \text { mortem }^{5} \text { and microglial activation } \\
\text { in vivo }{ }^{6} \text {. } \\
\text { Neurochemical dysfunction including elevated striatal dopamine } \\
\text { synthesis capacity? }\end{array}$ \\
\hline $\begin{array}{l}\text { A temporal relationship between the development of the } \\
\text { underlying disease and the onset of the mental syndrome }\end{array}$ & $\begin{array}{l}\text { There is a temporal relationship between the development of structural }{ }^{8} \\
\text { and neurochemical abnormalities }{ }^{7} \text { and the onset of psychosis . }\end{array}$ \\
\hline $\begin{array}{l}\text { Recovery from the mental disorder following removal or } \\
\text { improvement of the underlying presumed cause }\end{array}$ & $\begin{array}{l}\text { Schizophrenia is associated with raised striatal dopamine synthesis and } \\
\text { release capacity }{ }^{7} \text { and symptoms of schizophrenia improve when this is } \\
\text { blocked with dopamine antagonists. }\end{array}$ \\
\hline $\begin{array}{l}\text { Absence of evidence to suggest an alternative cause of the } \\
\text { mental syndrome such as a strong family history or } \\
\text { precipitating stress. }\end{array}$ & $\begin{array}{l}\text { This criterion is not appropriate. "Organic" illnesses can be hereditary } \\
\text { or contributed to by stress (e.g. hypertension). }\end{array}$ \\
\hline
\end{tabular}

The organic nature of schizophrenia has long been hypothesised. A century ago Emile Kraepelin suggested that we would one day be able to elucidate the neuropathology of dementia praecox (what we would now call schizophrenia). ${ }^{9}$ It took some time to develop the tools to investigate this in vivo, but many studies have now tested this.

In 1976 Johnstone et al. ${ }^{2}$ were the first to demonstrate, using computed tomography (CT), that patients with schizophrenia have increased lateral cerebral ventricle size. These results have since been replicated multiple times and further evidence of additional structural brain changes have been consistently found ${ }^{3}$. Moreover, brain imaging shows promise in the differential diagnosis of schizophrenia and mood disorders ${ }^{10}$. Structural changes have also been shown to pre-exist the first episode of psychosis, leading to the hypothesis that schizophrenia is a neurodevelopmental disorder ${ }^{8,11}$. Additionally, a meta-analysis using diffusion tensor imaging (DTI) has shown reduction in connectivity of two separate networks of white matter tracts, hence disconnection between grey matter regions ${ }^{4}$. This provides an organic basis for the functional dysconnectivity, i.e. abnormal connections, observed clinically in terms of symptomatology and experimentally, across a range of measures, in schizophrenia.

Furthermore, a classically described marker of an organic disease process is the presence of inflammation. Postmortem studies have shown evidence of elevated microglial cell density in the brains of patients with schizophrenia, particularly in the frontal and temporal lobes ${ }^{5}$, and there is recent in vivo evidence supporting these findings in participants at ultra-high-risk of developing psychosis ${ }^{6}$. These findings pre-date the onset of 
frank psychosis, suggesting causal association of the inflammatory activity and psychosis, and hence organic pathology.

In view of all this evidence, the fundamental criteria for organicity are either met in schizophrenia, or appear questionable (table). We can no longer divide illness into organic and inorganic based on the technological limitations of late $19^{\text {th }}$ Century anatomy and histology. Moreover, the current classification implies that there is a non-organic aetiology to schizophrenia, and by extension that this should direct management. Whilst it is clear that psychological and social factors play a major role in the illness, no psychological or social mechanism has been established as solely underlying the disorder or present in all cases, just as is the case for biological factors ${ }^{11}$. Instead, in most patients, a combination of psychological, social and biological factors play a role in the illness and its treatment, just as is the case with other common medical conditions such as diabetes mellitus.

The current classification of schizophrenia as a non-organic illness influences service organisation and resource allocations. Mental health services continue to be mostly separate from the rest of medical services, with distinct hospitals, teams and management. Moreover, in many countries, per capita spending on healthcare for patients with psychiatric illnesses is below that for non-psychiatric disorders. Already within an under-resourced sector, in the English National Health Service mental illnesses are further categorised into "non-psychotic", "psychotic" and "organic" illnesses for the purposes of resource allocation. This out-dated and trichotomous categorisation continues to be used in clinical decision-making and the commissioning and payment of services whereby patients are "clustered" and receive a "package of care" with a set tariff based on which category they fall in rather than healthcare need (https://www.gov.uk/government/uploads/system/uploads/attachment_data/file/300864/Guidance to mental_he

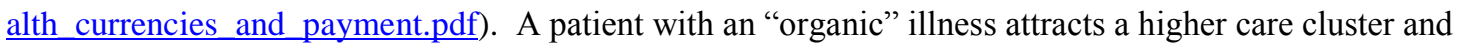
different payment tariff irrespective of actual needs. Despite decades of research indicating that this categorisation is inappropriate it continues to dominate resource allocation, with negative consequences for patients. 
The suggestion of a non-organic aetiology of mental illness also has an impact on its perception by the public and the rest of the medical profession. Despite psychiatry being a medical speciality, patients with schizophrenia are referred to psychiatrists from other specialists with comments in their clinical notes including that they are "medically fit" and have "no evidence of organic illness". This perpetuates a Cartesian dualistic fallacy that schizophrenia, and by extension other psychiatric illnesses, are quasi-illnesses that do not require the full range of healthcare available. Despite mental illnesses contributing a growing proportion of the disease burden, spending accounts for comparatively small proportions of clinical care, public health and medical research. For example, every year the UK Government invests approximately $£ 61$ per patient affected in research into psychotic illnesses, compared to $£ 1,571$ per patient for cancer $^{12}$.

Given the evidence for organic involvement in schizophrenia, and the implications of the non-organic distinction, it remains unclear why schizophrenia continues to be categorised as a non-organic illness despite evidence to the contrary. Perhaps refusal to accept the accumulated data might offer evidence for another clinical construction with its roots in the $19^{\text {th }}$ century: the psychoanalytic concept of denial?

\section{Contributors}

MAPB and ODH conceptualised the paper. All authors contributed to the intellectual content, critically edited, and approved the final version of the manuscript. 


\section{References}

1. World Health Organization. The ICD-10 classification of mental and behavioural disorders: clinical descriptions and diagnostic guidelines. Geneva: World Health Organization; 1992.

2. Johnstone EC, Crow TJ, Frith CD, Husband J, Kreel L. Cerebral ventricular size and cognitive impairment in chronic schizophrenia. Lancet 1976; 2(7992): 924-6.

3. Wright IC, Rabe-Hesketh S, Woodruff PW, David AS, Murray RM, Bullmore ET. Meta-analysis of regional brain volumes in schizophrenia. Am J Psychiatry 2000; 157(1): 1625.

4. Ellison-Wright I, Bullmore E. Meta-analysis of diffusion tensor imaging studies in schizophrenia. Schizophrenia research 2009; 108(1-3): 3-10.

5. Bayer TA, Buslei R, Havas L, Falkai P. Evidence for activation of microglia in patients with psychiatric illnesses. Neuroscience letters 1999; 271(2): 126-8.

6. Bloomfield PS, Selvaraj S, Veronese M, et al. Microglial Activity in People at Ultra High Risk of Psychosis and in Schizophrenia: An [(11)C]PBR28 PET Brain Imaging Study. Am J Psychiatry 2016; 173(1): 44-52. 
7. Howes OD, Kambeitz J, Kim E, et al. The nature of dopamine dysfunction in schizophrenia and what this means for treatment. Archives of general psychiatry 2012; 69(8): $776-86$.

8. Pantelis C, Velakoulis D, McGorry PD, et al. Neuroanatomical abnormalities before and after onset of psychosis: a cross-sectional and longitudinal MRI comparison. Lancet 2003; 361(9354): 281-8.

9. Kraeplin E. Dementia praecox and paraphrenia. Chicago: Chicago Medical Book Co; 1919.

10. Koutsouleris N, Meisenzahl EM, Borgwardt S, et al. Individualized differential diagnosis of schizophrenia and mood disorders using neuroanatomical biomarkers. Brain. 2015; 138: 2059-73

11. Howes OD, Murray RM. Schizophrenia: an integrated sociodevelopmental-cognitive model. Lancet 2014; 383(9929): 1677-87.

12. MQ Transforming Mental Health. Mental Health Research Funding Landscape Report. London: MQ Transforming Mental Health; 2015. 


\section{Conflict of Interest Statement}

Dr Bloomfield has conducted research funded by the Medical Research Council (UK), the National Institute on Health Research (UK) and the British Medical Association.

Dr Howes is supported by the Medical Research Council (UK), the Maudsley Charity and the National Institute for Health Research (NIHR) Biomedical Research Centre at South London and Maudsley NHS Foundation Trust and King's College London. Dr Howes has received investigator-initiated research funding from and/or participated in advisory/speaker meetings organized by Astra-Zeneca, BMS, Eli Lilly, Jansenn, Lundbeck, Lyden-Delta, Servier, and Roche. Neither Dr Howes nor his family have been employed by or have holdings/a financial stake in any biomedical company

Dr Buck has no financial conflict of interest to declare.

\section{Role of the funding source}

The funders had no role in the development of this paper. 